\title{
Recognition of odors effusing from living leaves using a SAW sensor system
}

\author{
Cai Kuiqian, Non-member (Faculty of Engineering, Toyama University)
}

Toyomasa Hatakeyama, Member (Faculty of Engineering, Toyama University)

Seichi Rengakuji, Non-member (Faculty of Engineering, Toyama University)

\begin{abstract}
Summary
An odor sensing system based on a SAW device has been developed for the measurement of odors from herb, which is a common name of the grass effusing relatively strong odors, and recognition of eight kinds of herb odors was performed using the system. Four kinds of lipid films were used as odor sensitive film in the study. The lipids of the films are (1) lecithin from eggs, (2) sphingomyelin, (3) L- $\alpha$-phosphatidyl ethanolamine solution $70 \%+$ phosphonicacid dioleyl ester $30 \%$ and (4) cholesterol $70 \%+$ sphingomyelin $30 \%$. The lipids were applied to the SAW resonator surface by spin coating, at speed 3000 r.p.m. for 20 seconds, from a lipid solution of $20 \mu \mathrm{l}$. A neural network algorithm was applied to identify the odors. In the recognition process, patterns consisting of maximum frequency changes of the sensors and differential frequency changes of the sensors between adsorption and desorption of the odors were taken as inputs of the network, and the patterns were normalized by a specific normalization method, so that the recognition of the odors was improved and done at rate of $99 \%$.
\end{abstract}

key words : SAW, herb, lipid, neural network, pattern normalization

\section{Introduction}

The importance of utilization of odors in various fields, for example, food processing and cosmetic industry, is getting more and more concerned with the functions of odor substance in recent years. Therefore, it is necessary to evaluate odors objectively using sensors instead of judging odors subjectively by human senses. Particularly, the functions of the plant odors, such as aromatherapy, sterilization and so on, have been studied actively.

Over the last fifteen years, the studies in development of the olfactory sensor have made much progress, with various types of sensors, that is, semiconductor gas sensors [1], sensors based on quartz resonators and surface acoustic wave (SAW) devices ${ }^{[2] \cdot[7]}$, conductive polymer sensors [B] and so on. Generally, the semiconductor gas sensors are of high sensitivity for a specific gas, but lack choice in the selectivity of many kinds of odor gases. The conductive polymer sensors are still in the early stage of the study. On the other hand, the sensors based on the quartz resonators and the SAW devices have various choices for the detection of various odor gases, and the frequency shift caused by mass loading in this type of sensors is proportional to the square oscillation frequency of the devices. Moreover, the SAW devices with its small size, ruggedness, high resonance frequency and low cost, are drawing public attention as transducers of chemical sensors.

The first study related to the use of the SAW devices as gas sensors were reported by Wholtjen and Dessy in $1979{ }^{[4]}$. Since then, many studies on olfactory sensors based on the SAW devices have been reported, and most of the reports were concerned with detection and recognition of odors from liquors, simple aromatics and so on. This paper introduces an olfactory SAW sensor system developed for odors from living herb leaves which have not been reported in the studies on those of sensors so far, although the functions of the herb odors have been widely studied in many fields.

\section{SAW sensors}

In the early stage of the study, we investigated the sensitivity of Tin oxide gas sensors, which are popular and commercially available, to the herb odors, and the sensors showed little sensitivity to the odors. In contrast with that, the sensors based on the SAW device had a variety 
of choice for the herb odors with relatively high sensitivity. Therefore, the SAW resonators were used as transducers for the odor sensor. An experiment was done to compare signal-to-noise ratio (SN ratio) with two types of resonators. The SN ratios were 300, 170 and 80 for the one-port type of resonator with $99.3 \mathrm{MHz}$, the one-port type of resonator with $211 \mathrm{MHz}$ and the two-port type of resonator with 224 $\mathrm{MHz}$, respectively, though there were about 3.5 times of the former's frequency shift on the shifts of the other resonators. The two-port type of resonator with $100 \mathrm{MHz}$ could not be obtained. Moreover, the oscillation circuits of the one-port type were simply made and had higher stability than that of two-port type. Consequently, we used one-port type of Y-X SAW resonator of $99.3 \mathrm{MHz}$ with R-SAW mode (SAR99MB41X MURATA MFG. Co., LTD) as the transducer.

Lipids show different responses for a kind of odor gas, and a certain lipid also performs differently for various odors. Paying attention to this phenomenon, we used four kinds of lipid films as adsorption materials. The lipids on the SAW resonators are (1) lecithin from eggs, (2) sphingomyelin, (3) L- $\alpha$-phosphatidyl ethanolamine solution $70 \%+$ phosphonicacid dioleyl ester $30 \%$ and (4) cholesterol $70 \%+$ sphingomyelin $30 \%$. The sensors coated with the lipids (1) to (4) are defined as S1, S2, S3 and S4 in the following section, respectively.

A frequency perturbation caused by changes in the thin film properties for a SAW device, such as mass loading, viscoelasticity and conductivity, can be described by the following equation [9];

$$
\Delta f=\left(k_{1}+k_{2}\right) f_{0}^{2} h \rho-\kappa_{2} f_{0}^{2} h\left[\frac{4 \mu}{v_{R}^{2}}\left[\frac{\lambda+\mu}{\lambda+2 \mu}\right]\right] .
$$

Where $k_{1}$ and $k_{2}$ are material constants of the substrate, $f_{0}$ is the resonance frequency of the oscillator, $h$ is the thickness of the film, $\rho$ is the density of the film, $\lambda$ is the Lame constant of the film, $\mu$ is the shear modulus of the film and $v_{R}$ is the Rayleigh wave velocity in the substrate. It should be noted that the first half of this equation is independent of the shear modulus (m) and that the quantity $(\lambda+\mu)$ / $(\lambda+2 \mu)$ is in the range 0.5 to 1.0 , with a value of 0.85 being typical for many polymeric materials. The shear modulusdependent portion of the equation becomes negligible when $4 \mu / v_{R}^{2}$ is less than 100 (i.e., for 'soft' polymers). Thus it is possible to write a simplified relationship between the resonance frequency of a SAW oscillator and mass loading properties.

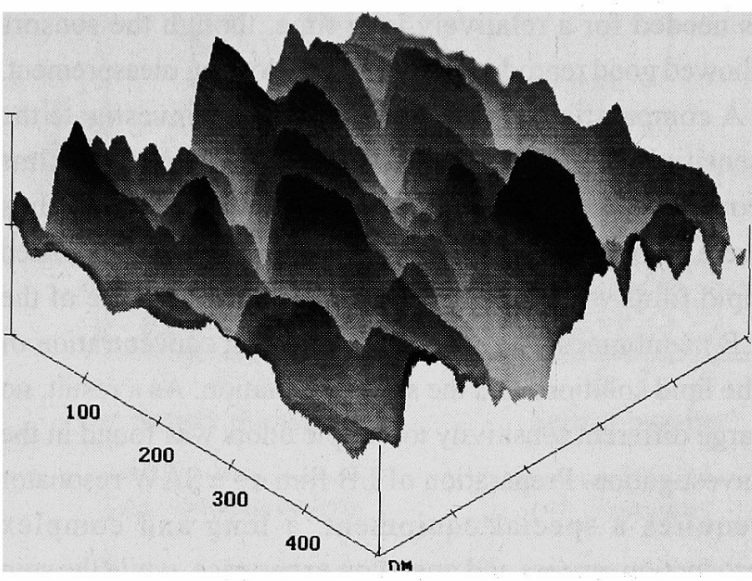

Fig.1 STM image of the surface for the SAW sensor coated with sphingomyelin.

$$
\Delta y=\left(k_{1}+k_{2}\right) f_{0}^{2} \frac{\Delta w}{A}
$$

where $\Delta w$ is the quantity of the mass loading, $A$ is the active surface area of the SAW device. The form of the function is in accordance with that of the function in the quartz resonator.

As an odor sensor, the SAW resonator is often separated from its circuit, so that stray capacitance, etc. easily affect the stability of the oscillation frequency. To keep stability of the oscillation frequency of the sensor, we used chip circuit parts for the oscillation circuit, and packed the sensors on sockets fixed inside of a sensor chamber. The pins of the sockets were directly connected to the circuit board. The sensor frequency drifts were less than about 5 $\mathrm{Hz}$ for 15 minutes when there is enough time for the measurement of the odors. The production of the lipid films was done by means of spin coating. The lipids were applied to the SAW resonator surface by spin coating, at speed 3000 r.p.m. for 20 seconds, from a lipid solution of $20 \mu 1$, which were diluted with chloroform at concentration 1:300, causing frequency shifts in the resonance frequency about $36 \mathrm{kHz}$.

Fig. 1 shows the surface of the sensor coated with sphingomyelin. The surface has a large active area through which the odor gas can diffuse and interact with a great number of sorption sites, obtaining a large sensor response for the odors [9]. On the other hand, it can also be considered that some of odors are diffused into a deeper layer of the film. The phenomenon could be seen in the first 
measurement for the odors in which the sensor's refreshment is needed for a relatively long time, though the sensors showed good reproducibility in the following measurement.

A comparative experiment was made to investigate the sensitivity of the SAW sensors with LB films and films coated by the spin coating. Hydrophobic lipid LB films were prepared with LB Trouph (SKV co.). The spin coated lipid films with the same frequency shifts as those of the LB membranes, were made by changing concentration of the lipid solutions and the speed of rotation. As a result, no large different sensitivity to sample odors was found in the investigation. Preparation of LB film on a SAW resonator requires a special equipment, a long and complex production process and operation experience, while the spin coating could be done simply with almost the same sensitivity as that of LB film for the sample odors in the experiment. Therefore, the spin coating method can be considered suited in practical use in this case.

\section{An olfactory sensing system}

An odor-sensing system with the SAW sensors mentioned above was constructed in the first stage of the study. Fig.2 shows a schematic diagram of the sensor system. The system consists of four SAW sensors with oscillation circuits, a high frequency relay circuit, a frequency counter, a GP-IB interface and a personal computer. The preparation of the samples was done as follows: Having picked up $1.5 \mathrm{~g}$ of living leaves of the herb, which were on third node from the top of stem, put it in a volume of $6 \mathrm{ml}$ space in the middle of the test tube. The top and bottom of the space was enclosed using stainless steel nets with silicon rubber rings on its frame.

During the measurement of the odors, $\mathrm{N}_{2}$ gas was introduced to the bottom of the test tube through a stainless steel needle at the flow rate of $100 \mathrm{ml} / \mathrm{min}$. and the odor gas was carried from the top of that into the sensor chamber. Oscillation frequencies of the sensors decrease with the quantity of the odor gases adsorbed on the sensor surfaces. Each of the sensors frequency shifts were inputted into the frequency counter every second, through the relay circuit, and then transferred to the computer. Those operations were controlled and performed automatically by the computer.

The oscillation frequency of the SAW sensors and defusing of the odors from the samples are changeable by its environmental temperature. The temperature of the measuring environment was controlled by an air conditioner, and the sensor chamber and the test tube with the living leaves were put in boxes made of expanded plastic to keep stable temperature at $25^{\circ} \mathrm{C} \pm 0.1^{\circ} \mathrm{C}$. The

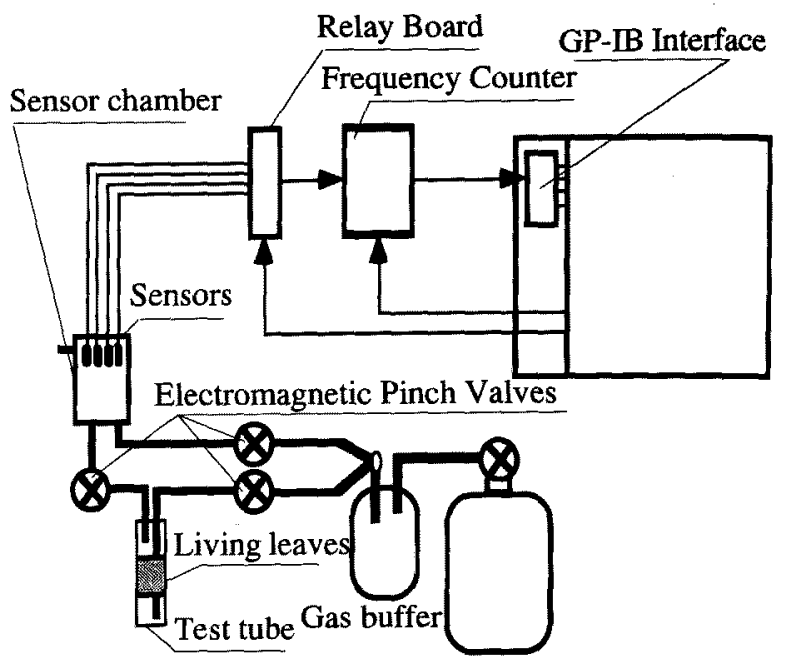

Fig.2 A schematic diagram of the SAW sensor system.

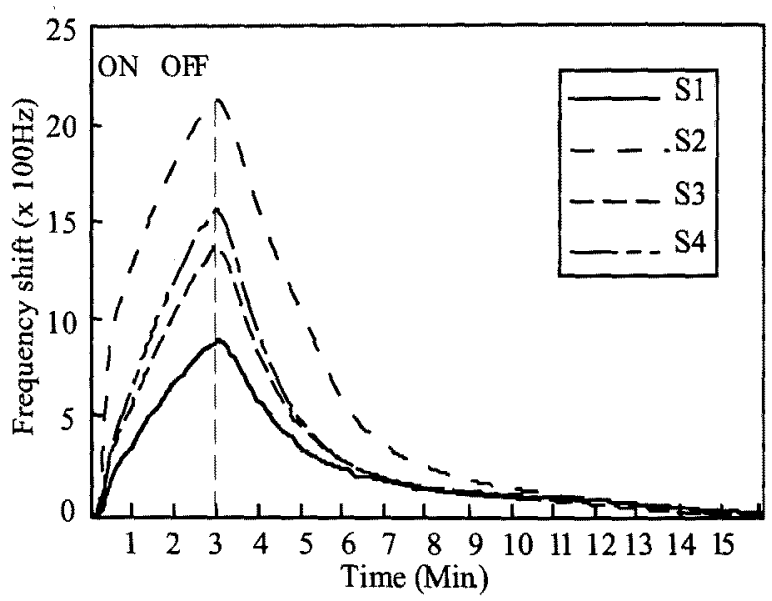

Fig.3 Sensor signals from the four sensors.

temperature coefficients of the SAW oscillation circuits of the sensors around $25^{\circ} \mathrm{C}$ were less than $5 \mathrm{ppm} /{ }^{\circ} \mathrm{C}$, therefore, the frequency changes in adjacent the temperature were very small, and so the measurement could be done with reproducibility.

Fig. 3 shows output signals from the four sensors for S.elegans. The vertical axis and the horizontal axis indicate a frequency shift in $\mathrm{Hz}$ and measuring time in minute after supplying the odor gas into the sensor chamber, respectively. The resonating frequencies of the sensors decreased with the amount of the odor gas adsorbed on the sensor surfaces. The supply of the odor gas was stopped to refresh the sensors for 15 minutes after supplying the odor 
gas for 3 minutes. In our sensing system the adsorption and the desorption of the odor gases were performed repeatedly to extend the life span of the sensors, in addition to the sensing process and avoiding over-adsorption of odors. The sensors could have been utilized for more than 6 months.

The sorts of the odors were discriminated using a neural network algorithm. The data measured in the first measurement was unstable, therefore, they were not taken into account in the recognition process of the odors. The similar result had been reported by Ide et al. in an odor sensing system ${ }^{[10] .}$

\section{Recognition of the odors using neural network}

As one of the ideology for the recognition of an odor by information process at level of the central nerves on living systems, the system identifies odors using the combination of the response patterns and the nerves information processing. On the other hand, sensor responses encode chemical information concerning each odor in a numerical form. Each sensor defines an axis of a coordinate system in space. The dimension of the coordinate space depends on the number of sensors used. Each odor can be thought of as a point whose position in the space is defined by the values produced by each sensor. Pattern recognition methods investigate such data clustering, and elucidate relationships in multidimensional data sets. The neural network algorithm, which is designed for the purpose of the application of neural information processing to industrial processes, is one of the widely used pattern recognition methods with its nonlinear data processing mechanism. Because of the nonlinearity of the sensor outputs for odors, the neural network algorithm is suitable for the sensor output processing and discriminations of sorts of odors, and the algorithm was used in our study.

Fig.4 shows the patterns which consist of maximum frequency changes of the four sensors normalized by the length of the vectors, for 8 kinds of the herb odors. For the different herb odors, the sensor outputs showed different patterns in Fig.4, though the patterns (4) and (5), and the patterns (7) and (8) were similar to each other, respectively. In order to increase the separation of the patterns another pattern normalization was performed in our recognition process. The normalization of pattern has three steps. Firstly, choose a value $\mathrm{N}$ that is a little larger than the maximum length of the vectors $\vec{E}_{i}^{\prime}\left(e_{i l}, e_{i 2}, e_{i 3}, e_{i 4}\right)$. Where number $i$ is related to the number of the herb odors in Fig(4), $e_{i j}$ is the maximum frequency shift of the $j$ th sensor output for the i th herb odor. Secondly, calculate values $d_{i}$ with
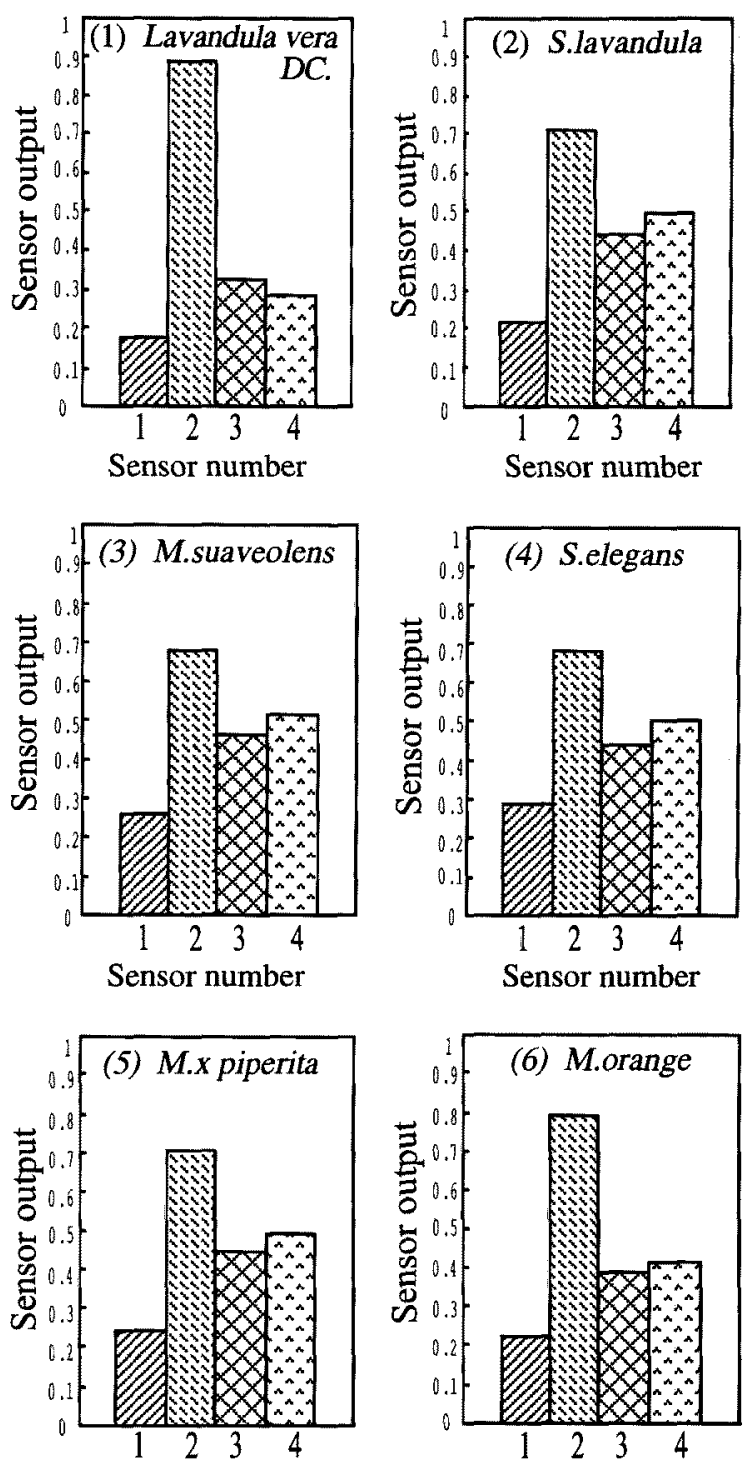

Sensor number

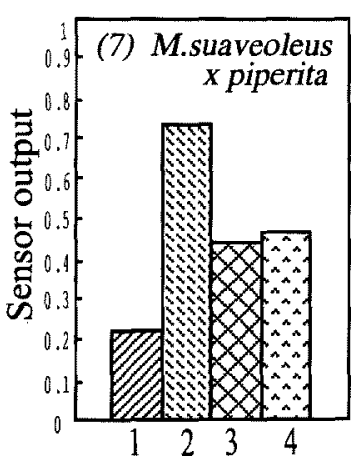

Sensor number

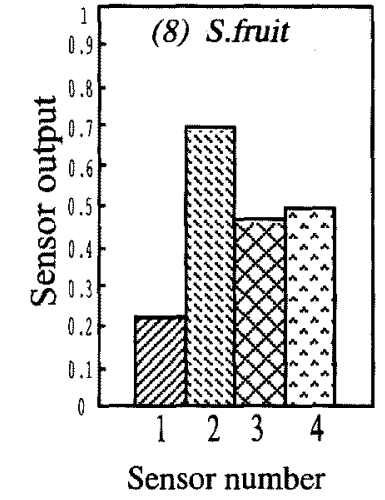

Fig.4 Sensor output patterns consisted of maximum frequency shifts for the eight kinds of herb odors. Sensor numbers are corresponded to the sensors coated with the lipids (1) to (4) mentioned in section 2. 
the function

$$
d_{i}=\sqrt{\left(N^{2}-\left\|\vec{E}_{i}^{\prime}\right\| \|^{2}\right)},
$$

and make new vectors $\vec{E}_{i}$,

$$
\vec{E}_{i}^{\prime \prime}\left(e_{i 1}, e_{i 2}, e_{i 3}, e_{i 4}, d_{i}\right)
$$

Finally, normalize the new vectors by the equation;

$$
\vec{E}_{i}=\frac{\vec{E}_{i}^{\prime \prime}}{N}
$$

Fig.5 shows the patterns normalized by the method mentioned above for M.x piperita and S.elegans. The numbers, 1 to 5 in Fig.5, are corresponded to S1, S2, S3, S4 and value $d$ calculated with the equation (3), respectively. In contrast with that the two patterns (4) and (5) in Fig.4 are similar to each other, the patterns in Fig. 5 are definitely different. On the other hand, abstracting more information from the olfactory sensors can also extend the separation of the data categories, and a kind of method using the gradient of sensor output is often applied in this case. However, the patterns consist of sensor output gradients in the odor adsorption and desorption were lack of reproducibility and had small changes for the different odors in our case. As compared with that, the differential frequency changes between adsorption and desorption of the odors were distinct for the every odors, and the patterns consist of those of differential value showed better reproducibility with larger distinction than the patterns consist of gradients of sensor outputs. Therefore, we further added the patterns, normalized by the equation (3)-(5), to the network as input data, in company with the other patterns mentioned above.

Fig.6 shows differential value of the frequency shifts between odor adsorption and desorption. The differential value is defined as follow:

$$
\Delta y=f_{A}-f_{B}
$$

Where the value $f_{A}$ is the sensor output after supplying the odor for 2 minutes and the value $f B$ is the sensor output after refreshing the sensors for 2 minutes. Consequently, the categories of the data for the odors could be separated distinctly by this process, increasing information from the time serial data of the sensor outputs, and so the rate of the recognition for the odors was improved. The network designed for the recognition of the herb odors has ten units for input layer and eight units for output layer which corresponds to the number of the herb odors to be measured. For the discrimination of the sample odors, we assigned

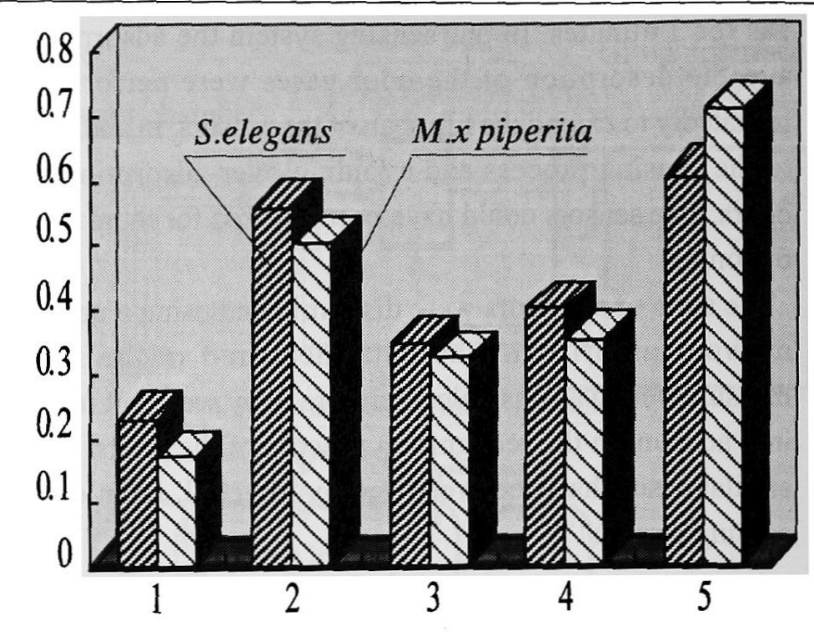

Fig.5 Ptterns after normalized by the new method.

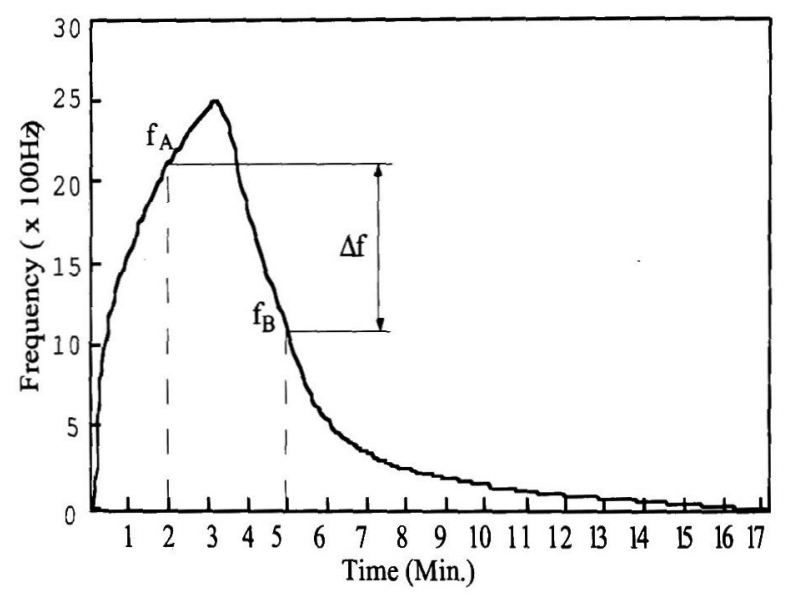

Fig.6 Differential value $(\Delta f)$ of the frequency shifts between

\begin{tabular}{|c|c|c|c|c|c|c|c|c|}
\hline \multirow{2}{*}{$\begin{array}{l}\text { Sample } \\
\text { Odors }\end{array}$} & \multicolumn{8}{|c|}{ Output Units } \\
\hline & 1 & 2 & 3 & 4 & 5 & 6 & 7 & 8 \\
\hline 1 & 8 & 0 & 0 & 0 & 0 & 0 & $\overline{0}$ & 0 \\
\hline 2 & 0 & 8 & 0 & 0 & 0 & 0 & 0 & 0 \\
\hline 3 & 0 & 0 & 8 & 0 & 0 & 0 & $\overline{0}$ & 0 \\
\hline 4 & 0 & 0 & 0 & 7 & 1 & 0 & 0 & $\overline{0}$ \\
\hline 5 & 0 & 0 & 0 & 0 & 8 & 0 & 0 & 0 \\
\hline 6 & 0 & 0 & 0 & 0 & 0 & 8 & 0 & $\overline{0}$ \\
\hline 7 & 0 & 0 & 0 & 0 & 0 & 0 & 8 & $\overline{0}$ \\
\hline 8 & 0 & 0 & 0 & 0 & 0 & 0 & 0 & 8 \\
\hline
\end{tabular}
odor adsorption and desorption.

Tab.1 Result of the recognition for the eight kinds of herb odors. 
each unit in the output layer to a specific sample odor. For example, when S.elegans odor is supplied and measured, assigned that the first unit has value 1 and the other units have value 0 in the output layer. This network signal state was taken as the training data in the training process. The network trained 5000 times with the error back propagation rule, using four sets of data that were randomly selected from 12 sets of data measured in practice. The recognition of the odors was performed with the rest of the eight sets of the data.

Tab. 1 shows the result of the identification of the odors effusing from the herb leaves. Where the odors are listed, in their numbers, in the first column, and output unit numbers corresponded to sorts of the odors are listed in the first row. If all the elements on the diagonal line of Tab.1 have value 8 , the recognition rate is $100 \%$. Although the recognition rate has not reached $100 \%$ in the study, the classification of the herb odors performed at about $99 \%$ rate. Applying pattern normalization method and increasing information from the sensors contributed to the increase of separation of categories of the herb odors so that the recognition rate was improved.

\section{Conclusion}

The SAW resonators as odor sensors are attractive, because of their high resonance frequency, small size, ruggedness, low cost and adaptability to a variety of odors with different adsorption films. In this study, we performed the recognition of odors from the 8 kinds of the living herb leaves using a SAW sensor system. The results of the study are given here as follows:

1. The SAW sensors had enough sensitivities to the living herb leaves.

2. The sensor system could be worked with acceptable reproducibility.

3. The frequency shift by spin coating for the same coatings were roughly equal to each other.

4. The unstable sensor outputs were appeared only in the first measurements, so that the first measurement data was not taken into account in the recognition of the odors to keep stabilities of the data.

5. The sensors life span could be more than 6 months.

6. A pattern normalization and differential frequency changes were used to enlarge the separation of the odor categories, and so improved the recognition rate.

In the future, we will make a comparative experiments for the differences between odors from living herb leaves and essential oils extracted from those of leaves using the sensing system for the purpose of the analytical use of the sensor system for herb plant odors. Furthermore, we will develop SAW sensor systems for monitoring the sound states of a plant at the outdoors and a tissue culture in a plant box.

(Manuscript received June 12, 1997, revised Oct. 13, 1997)

\section{Acknowledgment}

The authors would like to express many thanks to MURATA MFG. CO. LTD for offering us SAW elements.

\section{References}

[1] Persaud. K. and Dodd, G.: Analysis of discrimination mechanisms in the mammalian olfactory system using a model nose. Nature, 299; 352-355, 1982.

[2] T. Nakamoto and T.Moriizumi: Odor sensor using quartz-resonator array and neural-network pattern recognition, Proc. IEEE Ultrason. Symp.(IEEE, New York, 1988) Vol. 1, pp. 613.

[3] Y. Okahata and O. Shimizu: Olfactory reception on a multibilayer-coated piezoelectric crystal in a gas phase, Langmuir 3 (1987) 1171.

[4] H. Wholtjen and R. Dessy: Surface Acoustic Wave Probe for Chemical Analysis. I. Introduction and Instrument Description, Anal. Chem., vol.51, no.9, pp.1458-1464, Aug. 1979.

[5] Toyosaka Moriizumi, Atsushi Saitoh, Tooru Nomura: Partial Casting Method On Water Surface and Preparation of Multi-Channel SAW Chemical Sensor, Mol. Cryst. Lig. Cryst. 1994, Vol.247, pp. 331-339.

[6] Michiro Ohnishi, Coe Ishimoto and Masahiro Aomi: Fundamental properties and characteristics of a gassensitive device, made of a surface acoustic wave device and Langmuir-Blodgett film, used in a recognition system for odoriferous gases, Jpn. J. Appl. Phys. Vol.33, pp. 59875997, 1994.

[7] Hongyuan $\mathrm{Xu}$, Tatsuya Matsuda, Takamichi Nakomoto and Toyosaka Moriizumi: Phospholipid Film Electro deposition of quartz resonator and its application to odor sensing, Jpn. J. Appl. Phys. Vol. 34, pp. 3914-3919, 1995. [8] Persand, K. C. K. C. and Travers, P.: Intelligent Instruments \& Computers, Elsevier Science Publishing, New York, July/Aug., 147-154, 1991.

[9] H. Wholtjen: Mechanism of operation and design considerations for surface acoustic wave device vapour sensors, Sensors and Actuators, 5, 307, 1984.

[10] Junichi Ide, Takamichi Nakamoto and Toyosaka 
Moriizumi: Sensing film characterization for $\mathrm{QCM}$ array in an odor sensing system, T. IEE Japan, Vol. 116-E, No. $6, ' 96$.

Cai Kuiqian (non-member) He received the B.E. degree

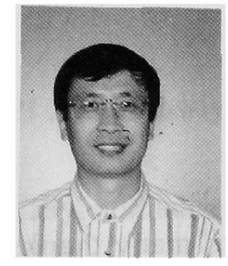
in mechanical engineering from Yanbian Agriculture College (now named as Yanbian University), China, in 1982 . He is currently in the doctor's course of the graduate school of Toyama University. $\mathrm{He}$ is interested in the olfactory sensor system based on SAW devices. He is a member of the Institute of Electronics Information and Communication Engineering.

Toyomasa Hatakeyama (member) He received the B.S.

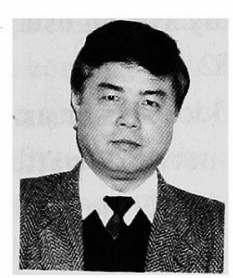
degree in physics from Toyama University in 1964, M.S. degree in physics from Kanazawa University in 1966 and D.E. degree in electrical engineering from Tokyo Institute of Technology in 1981 . He is currently a professor in Faculty of Engineering in Toyama University. His current research interest is a field related to bioelectricity such as biosensors, electric potentials from plants, electric effects for cell fusion and culture, and electrical properties of nucleic acids. He is a member of the Institute of Electronics Information and Communication Engineering, Japan Society of Applied Physics, the Society of Instrument and Control Engineers, and the Society for Fermentation and Bioengineering, Japan, respectively.

Seichi Rengakuji (non-member) He received a B.E. degree

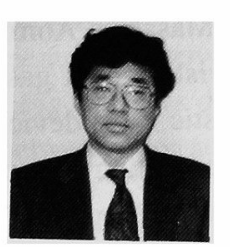
in 1975 from Toyama University, a Dr. of Eng. from Kanazawa University in 1995 . He is currently an assistant professor in Faculty of Engineering in Toyama University. He has been involved in the research of a surface improvement, energy storage system, and electrochemical system with LB film. 